\title{
Robust Gapless Surface State and Rashba-Splitting Bands upon Surface Deposition of Magnetic $\mathrm{Cr}$ on $\mathrm{Bi}_{2} \mathrm{Se}_{3}$
}

\author{
Eryin Wang, ${ }^{\dagger}, \mathrm{O}$ Peizhe Tang, ${ }^{\dagger, O}$ Guoliang Wan, ${ }^{\dagger}$ Alexei V. Fedorov, ${ }^{\ddagger}$ Ireneusz Miotkowski, ${ }^{\S}$
} Yong P. Chen, ${ }^{\S, \|, \perp}$ Wenhui Duan, ${ }^{*},, \Phi, \nabla$ and Shuyun Zhou* ${ }^{*},, \nabla$

\author{
${ }^{\dagger}$ State Key Laboratory of Low Dimensional Quantum Physics and Department of Physics, Tsinghua University, Beijing 100084, P.R. \\ China \\ ${ }^{\ddagger}$ Advanced Light Source, Lawrence Berkeley National Laboratory, Berkeley, California 94720, United States \\ ${ }^{\S}$ Department of Physics and Astronomy, Purdue University, West Lafayette, Indiana 47907, United States \\ "Birck Nanotechnology Center, Purdue University, West Lafayette, Indiana 47907, United States \\ ${ }^{\perp}$ School of Electrical and Computer Engineering, Purdue University, West Lafayette, Indiana 47907, United States \\ II Institute for Advanced Study, Tsinghua University, Beijing 100084, P.R. China \\ ${ }^{\nabla}$ Collaborative Innovation Center of Quantum Matter, Beijing 100084, P.R. China
}

ABSTRACT: The interaction between magnetic impurities and the gapless surface state is of critical importance for realizing novel quantum phenomena and new functionalities in topological insulators. By combining angle-resolved photoemission spectroscopic experiments with density functional theory calculations, we show that surface deposition of $\mathrm{Cr}$ atoms on $\mathrm{Bi}_{2} \mathrm{Se}_{3}$ does not lead to gap opening of the surface state at the Dirac point, indicating the absence of long-range out-of-plane ferromagnetism down to our measurement temperature of $15 \mathrm{~K}$. This is in sharp contrast to bulk $\mathrm{Cr}$ doping, and the origin is attributed to different $\mathrm{Cr}$ occupation sites. These results highlight the importance of nanoscale configuration of doped magnetic impurities in determining the electronic and magnetic properties of topological insulators.

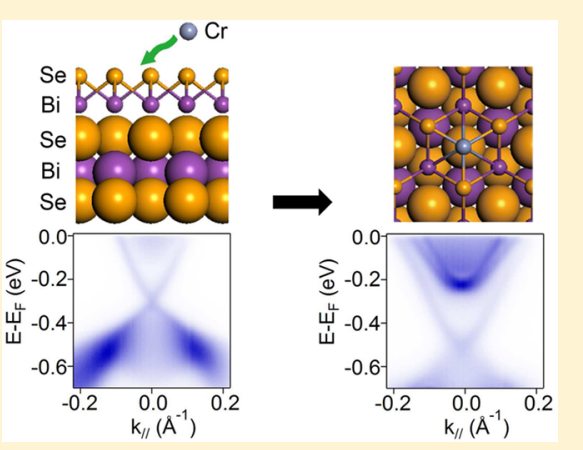

KEYWORDS: topological insulator, time-reversal symmetry, ferromagnetism, angle-resolved photoemission spectroscopy (ARPES)

$\mathrm{T}$ he three dimensional (3D) topological insulator (TI), a novel class of materials, ${ }^{1,2}$ is a topologically nontrivial bulk insulator with conducting surface state (SS). Protected by the time-reversal symmetry (TRS), the SS is spin-polarized, gapless, and robust against nonmagnetic impurities. ${ }^{3-6}$ Meanwhile, magnetic impurities may induce out-of-plane ferromagnetism and break the TRS in TI, resulting in a band gap at the Dirac point of the SS. ${ }^{7}$ Such TRS breaking is essential for the realization of various novel quantum phenomena, which have great application potential in future nanoelectronic and spintronic devices, such as quantum anomalous Hall effect (QAHE), ${ }^{8-11}$ topological magnetoelectric effect (TME), ${ }^{12}$ and birefringent spin lens. ${ }^{13}$ Thus, it is critical to understand the interaction between 3D TIs and magnetic impurities both for fundamental physics and nanodevice applications.

The interaction between $3 \mathrm{D}$ TI and doped magnetic impurities can modify the electronic and magnetic properties of 3D TI, and the results may strongly depend on the types of magnetic atoms, occupation sites of the magnetic impurities, ${ }^{14}$ and experimental conditions under which they are introduced. For example, although doping $\mathrm{Fe}$ and $\mathrm{Cr}$ into the bulk $\mathrm{Bi}_{2} \mathrm{Se}_{3}$ crystal or thin film during the growth process leads to a gap opening at the Dirac point suggesting TRS breaking, ${ }^{7,15-17}$ the robustness of the gapless $\mathrm{SS}$ against $\mathrm{Fe}$ deposition on the surface has been debated ${ }^{18,19}$ and surface deposition of Cr still remains to be investigated. Here, we present a combined experimental and theoretical study of the electronic structures of $\mathrm{Bi}_{2} \mathrm{Se}_{3}$ upon surface deposition of magnetic $\mathrm{Cr}$ atoms on freshly cleaved $\mathrm{Bi}_{2} \mathrm{Se}_{3}$ single crystals. Angle-resolved photoemission spectroscopy (ARPES) data show that the SS is robust upon surface deposition of $\mathrm{Cr}$, and the absence of gap opening at the Dirac point suggests that there is no long-range out-of-plane ferromagnetism down to the lowest measurement temperature of $15 \mathrm{~K}$. In addition, $\mathrm{Cr}$ atoms dope electrons to the bulk states of $\mathrm{Bi}_{2} \mathrm{Se}_{3}$ forming a two-dimensional electron gas with large Rashba-splitting on the surface. These observations are in striking contrast to the bulk doping of $\mathrm{Cr}$ during thin film growth, ${ }^{15}$ suggesting that $\mathrm{Cr}$ atoms deposited on the surface interact very differently with the TI sample. Density functional theory (DFT) calculations show that the difference in electronic and magnetic properties originates from the different $\mathrm{Cr}$ configurations: hollow site for surface deposition versus substitutional site for bulk doping. Due to the coupling between Cr $3 \mathrm{~d}$ orbitals around the Fermi level, the magnetic easy axis of

Received: December 21, 2014

Revised: February 14, 2015

Published: February 24, 2015 

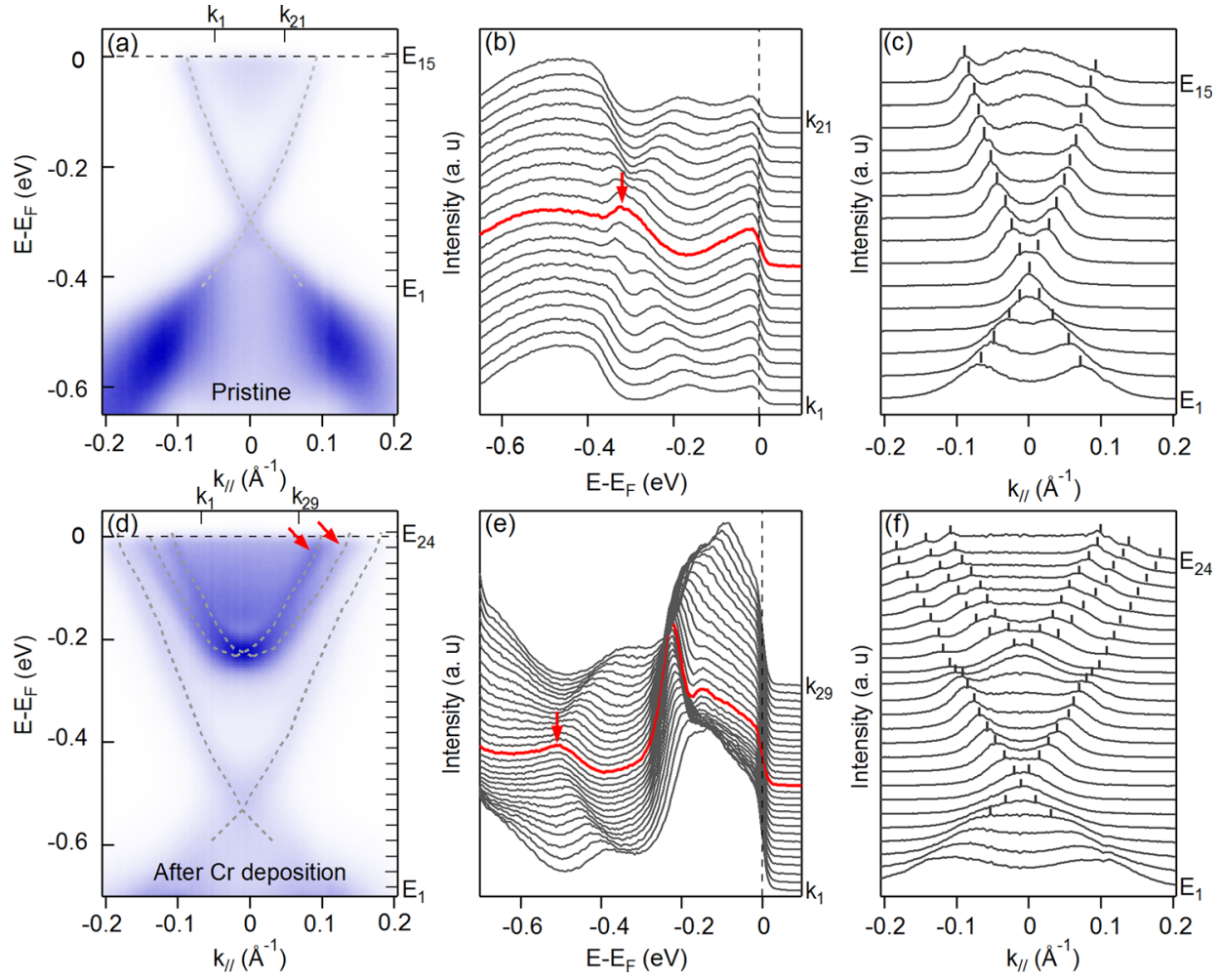

Figure 1. (Color online) (a) Dispersion image along $M-\Gamma-M$ direction. Dotted lines show dispersions extracted from MDCs in (c). (b) EDCs at momentum positions between $k_{1}$ and $k_{21}$ marked by ticks in (a). EDC at the $\Gamma$ point is highlighted by red color. (c) MDCs at energy positions marked by ticks between $E_{1}$ and $E_{21}$ in (a). Ticks are guides for peak positions of the SS. (d) Dispersion image along $M-\Gamma-\mathrm{M}$ direction after surface deposition of $\mathrm{Cr}$ for $46 \mathrm{~min}, \sim 9 \% \mathrm{ML} \mathrm{Cr}$ on $\mathrm{Bi}_{2} \mathrm{Se}_{3}$. Dotted lines show dispersions extracted from MDCs in (f). (e) EDCs at momentum positions between $k_{1}$ and $k_{29}$ marked by ticks in (d). EDC at $\Gamma$ point is highlighted by red color. (f) MDCs at energy positions marked by ticks between $E_{1}$ and $E_{24}$ in (d). Ticks are guides for peak positions of the SS and Rashba splitting bands.
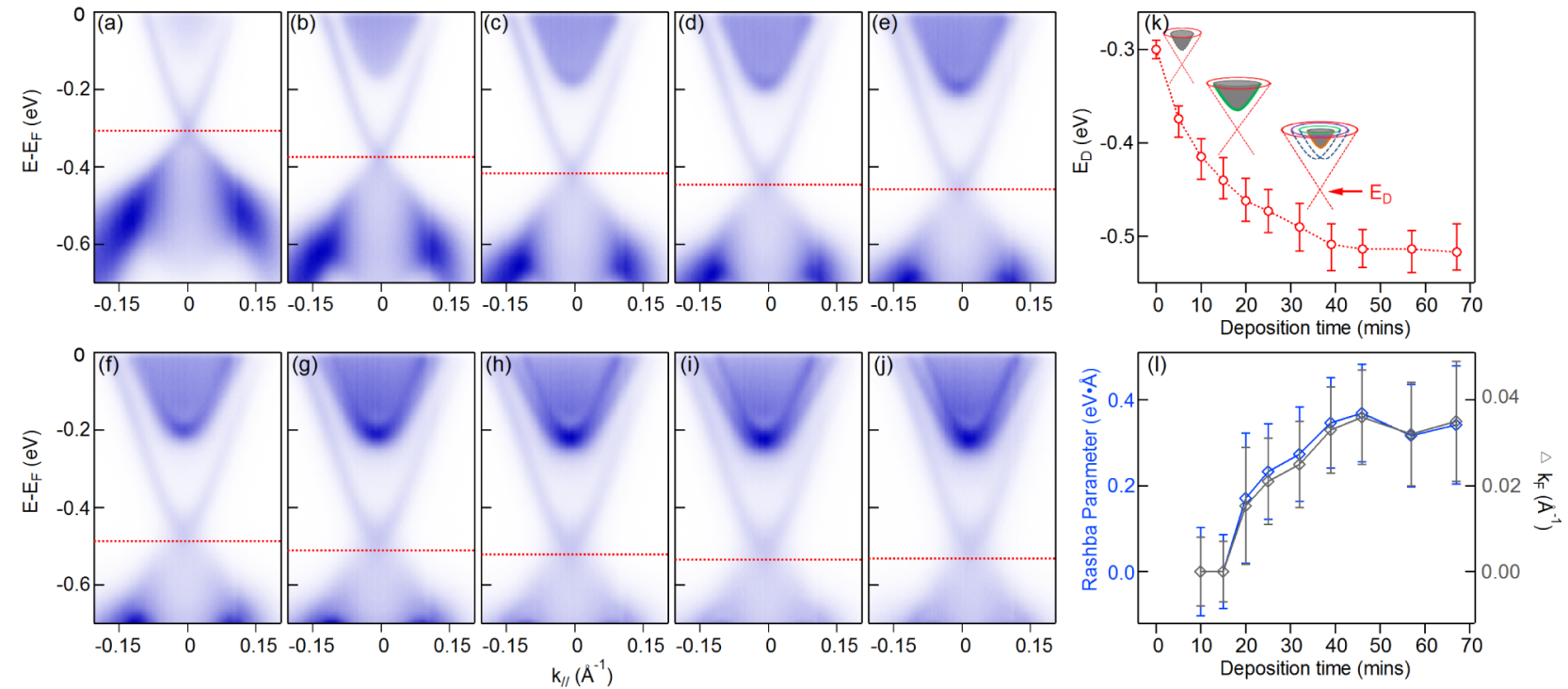

Figure 2. (Color online) (a-j) Dispersions along $M-\Gamma-M$ direction on pristine $\mathrm{Bi}_{2} \mathrm{Se}_{3}$ (a) and with different Cr deposition time $5,10,15,20,25$, $32,39,46$, and $57 \mathrm{~min}(\mathrm{~b}-\mathrm{j})$. Red dotted lines indicate the energy positions of the Dirac point. (k) Evolution of the Dirac point energy as a function of $\mathrm{Cr}$ deposition time. The inset shows a schematic illustration of the band structure evolution. (1) Rashba splitting $\Delta k_{\mathrm{F}}$ (black symbols, right axis) and calculated Rashba coupling parameter $\alpha$ (blue symbols, left axis) as a function of $\mathrm{Cr}$ deposition time.

deposited $\mathrm{Cr}$ atoms is likely along the in-plane direction, which is in agreement with the robustness of the SS. These results highlight the importance of nanoscale configuration of magnetic impurities in determining the electronic and magnetic properties of topological insulators, and such understanding is critical for manipulating the electronic properties of TI and future electronic applications.

Figure 1 shows a comparison of ARPES data measured at 15 $\mathrm{K}$ with $35 \mathrm{eV}$ photon energy on freshly cleaved $\mathrm{Bi}_{2} \mathrm{Se}_{3}(111)$ surface (a) and after deposition of $\mathrm{Cr}$ atoms on the surface for 
(c)

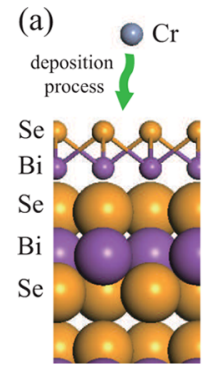

(b)

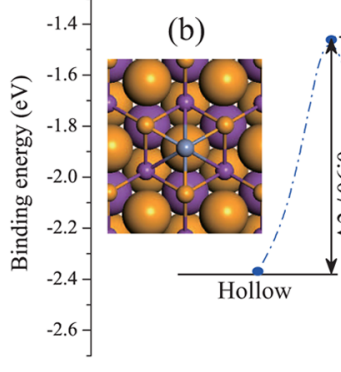

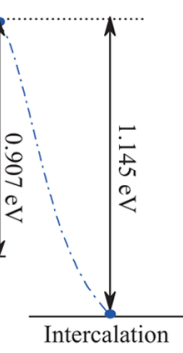

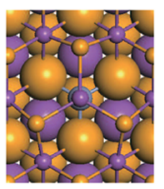

(d)

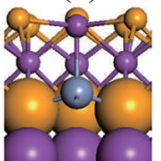

(e)

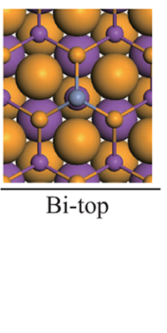

(f)

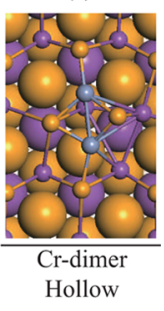

Figure 3. (Color online) (a) Schematic diagram showing the deposition process of $\mathrm{Cr}$ atom to the surface of $\mathrm{Bi}_{2} \mathrm{Se}_{3}$. (b-f) Binding energies of $\mathrm{Cr}$ absorption on different sites: (b) hollow; (c,d) intercalation; (e) Bitop; (f) Cr-dimer absorption on the hollow site. (b,c,e,f) are the top views and (d) is the side view. Black solid lines show the binding energies of absorption on different sites. The blue dash dot line stands for the chemical reaction path from climbing NEB calculation. The energy barrier is $0.907 \mathrm{eV}$.

46 min, with coverage $\sim 9 \%$ monatomic layer (ML) $\mathrm{Cr}$ (d). ARPES data on pristine $\mathrm{Bi}_{2} \mathrm{Se}_{3}$ [Figure 1a] shows conical dispersion from the SS with Dirac point energy at $-0.3 \mathrm{eV}$, a small parabolic bulk conduction band near $E_{\mathrm{F}}$. After deposition of $\mathrm{Cr}$ for $46 \mathrm{~min}$, the SS persists and is electron doped, with the Dirac point energy shifted down to $-0.51 \mathrm{eV}$. Energy distribution curves (EDCs) and momentum distribution curves (MDCs) analysis shows that there is no gap opening at the Dirac point of the SS down to $15 \mathrm{~K}$ [Figure 1e, $\mathrm{f}$ ]. This is in striking difference from bulk doping of $\mathrm{Cr}$ into $\mathrm{Bi}_{2} \mathrm{Se}_{3}$ films during MBE growth, ${ }^{15,20}$ where a significant gap at the Dirac point was reported at a much higher temperature. Despite the magnetic moment of $\mathrm{Cr}$ atoms, ${ }^{21-23}$ our results suggest the surface state is very robust upon $\mathrm{Cr}$ surface deposition.

While doping electrons to the SS with preserved TRS, Cr deposition also induces additional bands (pointed to by arrows in Figure 1d), which are attributed to two-dimensional electron gas (2DEG) formed on the sample surface. Figure $2 \mathrm{a}-\mathrm{j}$ show the evolution of the band dispersion with increasing $\mathrm{Cr}$ deposition time. Initial deposition induces electron doping to the sample and shifts the SS and bulk bands to higher binding energy. A parabolic band of $2 \mathrm{DEG}$ forms at the bottom of the bulk conduction band after depositing $\mathrm{Cr}$ for $10 \mathrm{~min}$ [Figure $2 \mathrm{c}$ ]. With increasing $\mathrm{Cr}$ deposition, the $2 \mathrm{DEG}$ band becomes more clear and starts to show momentum splitting [Figure $2 \mathrm{e}-$ j] due to the formation of effective potential gradient across the sample surface. Such momentum-displaced Rashba effect derives from the spin-orbit coupling due to the effective potential gradient on the surface, $(\vec{k} \times \hat{z}) \cdot \vec{\sigma}$, where $\vec{\sigma}$ is the Pauli matrices. The dispersion of the Rashba splitting bands can be approximated as $E^{ \pm}\left(k_{\|}\right)=E_{0}+\left(\hbar^{2} k_{\|}^{2} /\left(2 m^{*}\right) \pm \alpha \mid k_{\|} l\right.$, where $m^{*}$ is the effective mass and $\alpha$ is the Rashba coupling parameter. Similar effect has been observed for various gas absorption or metal deposition on $\mathrm{Bi}_{2} \mathrm{Se}_{3}$ surface, for example, $\mathrm{CO}^{24}$ and water, ${ }^{25}$ nonmagnetic alkaline metals. ${ }^{26}$ From the evolution of electronic structure with $\mathrm{Cr}$ deposition, we extract the energy position of Dirac point $E_{\mathrm{D}}$ [Figure $2 \mathrm{k}$ ] and the momentum splitting value $\Delta k_{F}$ [Figure 2l] after different deposition time. Comparing the evolution of $E_{D}$ and $\Delta k_{F}$, we can see the Dirac point shifts downward quickly before the 2DEG band starts splitting after around $20 \mathrm{~min}$ of $\mathrm{Cr}$ deposition. This implies the deposited $\mathrm{Cr}$ atoms first donate electrons to $\mathrm{Bi}_{2} \mathrm{Se}_{3}$ sample and then form $2 \mathrm{DEG}$ with increasing effective electrical potential near the sample surface. The amount of charge transfer can be calculated from the change in the Fermi surface area including the SS, outer and inner Rashba bands using Luttinger's theorem. ${ }^{27}$ The charge transfer of $\mathrm{Cr}$ atoms after $46 \mathrm{~min} \mathrm{Cr}$ deposition is estimated to be $q \sim 0.06 e^{-}$ per unit cell. The Rashba coupling parameter $\alpha$ can be extracted using $\alpha=\Delta k_{F} / 2 m^{*}$, where the $m^{*}$ is the effective mass of 2DEG. From Figure 2l, the maximum coupling parameter is $0.34 \mathrm{eV} \cdot \AA$, which is comparable with those of $\mathrm{Au}(111)$ surface $^{28,29}$ and 2 DEG in semiconductors. ${ }^{30,31}$

The absence of gap opening at the Dirac point and the emergence of Rashba-splitting bands suggest that the $\mathrm{Cr}$ atoms deposited on the surface interact with TI very differently from bulk doping. ${ }^{15}$ In order to understand why surface deposition of $\mathrm{Cr}$ atoms does not lead to the gap opening as in the case of bulk $\mathrm{Cr}$ doping, we use DFT to simulate the deposition process of $\mathrm{Cr}$ atoms on $\mathrm{Bi}_{2} \mathrm{Se}_{3}(111)$ surface. At the beginning of the simulation, we put $\mathrm{Cr}$ atoms on different initial positions (including intercalation site, hollow site, bridge site, Bi-top site, and Se-top site), and let them fully relax. It is found that $\mathrm{Cr}$ atoms on the Se-top site and bridge site will spontaneously relax to the Bi-top site. Therefore, we only have three energetically stable configurations as shown in Figure 3. They are $\mathrm{Cr}$ absorptions on the hollow site [Figure $3 \mathrm{~b}$ ], intercalation site [Figure 3c,d], and Bi-top site [Figure 3e], with binding energies $\left(E_{\mathrm{b}}\right)^{32}$ of $-2.378 \mathrm{eV},-2.631 \mathrm{eV}$, and $-2.200 \mathrm{eV}$ respectively. Because $E_{\mathrm{b}}$ of all optimal structures are negative, the energy will be released during the deposition process, and the structures with absorbed $\mathrm{Cr}$ atoms are stable. Although the configuration for $\mathrm{Cr}$ atom on the intercalation site has the lowest $E_{\mathrm{b}}$, this structure is practically difficult to be realized. According to the climbing nudged elastic band (NEB) calculation, $^{33}$ a large energy barrier (about $1 \mathrm{eV}$ ) should be overcome when $\mathrm{Cr}$ atom intercalates into the atomic layers underneath. Therefore, the most feasible configuration observed experimentally is $\mathrm{Cr}$ absorption on the hollow site with the relatively lower $E_{\mathrm{b}}$. This is very different from the substitutional Bi site for bulk $\mathrm{Cr}$ doping. ${ }^{15}$

Here, we focus on the electronic structure of $\mathrm{Cr}$ single absorption on the hollow site, and its density of state (DOS) is shown in Figure 4. Due to $C_{3 v}$ symmetry around the absorption site, the crystal field splits $C r 3 \mathrm{~d}$ orbitals into $A_{1}\left(\mathrm{~d}_{z^{2}}\right), E_{1}\left(\mathrm{~d}_{x z}\right)$ $\left.\mathrm{d}_{y z}\right)$ and $E_{2}\left(\mathrm{~d}_{x}^{2}-y^{2}, \mathrm{~d}_{x y}\right)$ states. Considering the Hunt's rule, a large energy splitting between the spin-majority states and the spin-minority states can be observed from our DFT calculations, and the five spin-majority states of $\operatorname{Cr}\left(A_{1}^{\uparrow}, E_{1}^{\uparrow}\right.$, and $E_{2}^{\uparrow}$ ) are fully occupied [see Figure $4 \mathrm{a}$ ]. Due to the similar in-plane orientation, a strong $\mathrm{p}-\mathrm{d}$ hybridization between Se $\mathrm{p}$ orbitals and $\mathrm{Cr} \mathrm{d}_{x^{2}-y^{2}}$ and $\mathrm{d}_{x y}$ orbitals drives $E_{2}^{\uparrow}$ states close to 

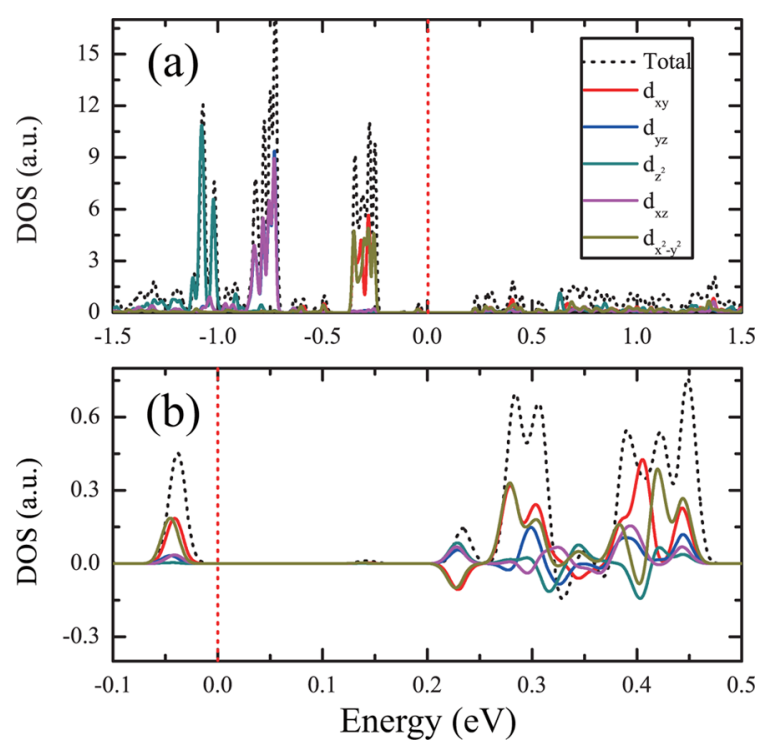

Figure 4. (Color online) (a) Total and orbital-projected density of states (DOS) of $\mathrm{Cr}$ atom for the single adsorption on the hollow site. (b) $\sigma_{x}$ component of DOS. The positive and negative stand for the spin-majority and spin-minority, respectively. The Fermi level is set to zero and marked by the red dot line.

the Fermi level, and the spin-polarized electron density becomes more extended which also could be found on the neighboring Se atoms. Therefore, the total magnetic moment of this structure is $5 \mu_{\mathrm{B}}$.

For magnetic properties of $\mathrm{Cr}$ absorption on the hollow site, we calculate the magnetic anisotropy energy (MAE) from DFT simulations. It is found the magnetic easy axis is along the inplane direction with MAE of $2.04 \mathrm{meV} /$ atom. Based on the perturbation theory, ${ }^{34,35}$ around the Fermi level, MAE is mainly determined by the difference of SOC matrix elements $\left(H_{i j}^{S O}(\hat{n})\right.$ $\left.\equiv\left\langle i\left|H^{S O}(\hat{n})\right| j\right\rangle\right)$ along in-plane and out-of-plane direction, which can be expressed as $\sum_{i, j}\left(1 /\left(\left.\Delta_{i j}\left(\left|H_{i j}^{S O}(\hat{x})\right|^{2}-\mid H_{i j}^{S O}(\hat{z})\right)\right|^{2}\right)\right.$, where $i$ and $j$ stand for the occupied and unoccupied states respectively and $\Delta_{i j}$ is their energy difference. Shown in Figure $4 \mathrm{~b}$, around the Fermi level, the states of $\mathrm{Cr}$ atom are mainly contributed by hybridized $\mathrm{d}$ orbitals with opposite spins, and the coupling between them results in an in-plane magnetic easy axis. ${ }^{35,36} \mathrm{In}$ TIs, the SS is protected by TRS and can only be gapped by outof-plane ferromagnetism ${ }^{8}$ or in-plane ferromagnetism combined with mirror symmetry breaking. ${ }^{37}$ The absence of gap opening at the Dirac point in the ARPES data is in agreement with the in-plane magnetic easy axis. ${ }^{19}$

If absorbed $\mathrm{Cr}$ atoms form in-plane ferromagnetism without breaking other symmetry, the effective exchange field will shift the Dirac point of SS without opening a band gap. The shift magnitude $\left(k_{\|}^{D}\right)$ can be estimated by $k_{\|}^{D} \equiv m_{\hat{x}} / v_{\mathrm{F}}$, in which $m_{\hat{x}}$ is the in-plane ferromagnetic coupling strength and $v_{\mathrm{F}}$ is the Fermi velocity of $\mathrm{Bi}_{2} \mathrm{Se}_{3}\left(v_{\mathrm{F}}=3.55 \mathrm{eV} \cdot \AA^{38}\right)$. To estimate the shift of Dirac point, in our view, the maximum magnetic coupling strength should have the same order of magnitude with that of $\mathrm{Cr}$-dimer absorbed on $\mathrm{Bi}_{2} \mathrm{Se}_{3}(111)$ surface. Considering $\mathrm{Cr}$-dimer absorption on the hollow site, calculated results are shown in Figure 3f: the distance between two absorbed $\mathrm{Cr}$ atoms is $2.87 \AA$ and $E_{\mathrm{b}}$ is $-2.409 \mathrm{eV}$. This value is slightly lower than that of single $\mathrm{Cr}$ absorption on hollow site [see Figure $3 \mathrm{~b}$ ], suggesting both structures can be observed in the experiment. The magnetic easy axis of $\mathrm{Cr}$ dimer is also along the in-plane direction with MAE of $3.32 \mathrm{meV} /$ atom, and two $\mathrm{Cr}$ atoms prefer ferromagnetic coupling with coupling strength of $69.21 \mathrm{meV}$, and the average magnetic moment of each $\mathrm{Cr}$ atom is about $3.2 \mu_{\mathrm{B}}$. If the in-plane ferromagnetic coupling formed in $\mathrm{Cr}$ deposited $\mathrm{Bi}_{2} \mathrm{Se}_{3}$ surface, using $k_{\|}^{D} \equiv m_{\hat{x}} /$ $v_{\mathrm{F}}$, we estimate the maximum momentum shift $k_{\|}^{D}$ is about 0.02 $\AA^{-1}$, which is within our experimental uncertainty. We note that the absence of long-range ferromagnetic order was reported ${ }^{19}$ in previous studies of surface deposition of $\mathrm{Fe}$ on $\mathrm{Bi}_{2} \mathrm{Se}_{3}$ surface. Here, we tend to believe that there is no long-range ferromagnetic order down to $15 \mathrm{~K}$ for surface deposition of $\mathrm{Cr}$, which needs to be confirmed by direct magnetic measurements.

In conclusion, our combined experimental and theoretical study show that, different from bulk $\mathrm{Cr}$ doped $\mathrm{Bi}_{2} \mathrm{Se}_{3}$, surface deposition of $\mathrm{Cr}$ atoms on $\mathrm{Bi}_{2} \mathrm{Se}_{3}$ shows no gap opening at Dirac point, implying that no out-of-plane ferromagnetism is formed on surface down to our measurement temperature of 15 $\mathrm{K}$. According to DFT calculations, the difference of electronic and magnetic properties originates from the different $\mathrm{Cr}$ configurations: surface deposition on the hollow site versus substitutional site for bulk doping. Due to the coupling between Cr 3d orbitals around the Fermi level, the magnetic easy axis of deposited $\mathrm{Cr}$ atoms is along the in-plane direction. In addition, deposited $\mathrm{Cr}$ atoms induce electron doping to the sample and form Rashba-splitting 2DEG bands near the sample surface. These results indicate the interaction between magnetic dopants and TIs strongly depends on the intricate configuration of magnetic atoms, which has important implication for manipulation of SS in TIs.

Methods. ARPES measurements were carried out at Beamline 12.0.1 of the Advanced Light Source with total energy resolution better than $25 \mathrm{meV}$. High quality bulk single crystal $\mathrm{Bi}_{2} \mathrm{Se}_{3}$ samples were cleaved in situ in UHV chamber with base pressure of $2.5 \times 10^{-11}$ Torr before the measurements. The sample was kept at $15 \mathrm{~K}$ during $\mathrm{Cr}$ deposition and ARPES measurements. Cr was evaporated from a resistively heated tungsten basket, and the flux was calibrated by a quartz microbalance to be $\sim 0.17 \AA$ per minute prior to experiment. Due to the different sticking coefficients between quartz and $\mathrm{Bi}_{2} \mathrm{Se}_{3}(111)$ surface, the actual amount of $\mathrm{Cr}$ atoms absorbed on $\mathrm{Bi}_{2} \mathrm{Se}_{3}$ surface cannot be obtained directly. In order to estimate the amount of $\mathrm{Cr}$ absorbed on $\mathrm{Bi}_{2} \mathrm{Se}_{3}$ surface, we compare the charge transfer from the Bader charge analysis ${ }^{39}$ in the DFT calculation with the experimental charge transfer. The Bader charge analyis shows each $\mathrm{Cr}$ atom transfers 0.361 electron to $\mathrm{Bi}_{2} \mathrm{Se}_{3}$ surface. Experimentlly, using Luttinger's theorem, ${ }^{27}$ the total charge transfer from $\mathrm{Cr}$ atoms calculated from the change of Fermi surface area after deposition for 46 min is 0.06 electron per unit cell. Thus, we estimate 0.06 / $0.361-1 / 6 \mathrm{Cr}$ atom was absorbed per unit cell after $46 \mathrm{~min}$ deposition, and the corresponding coverage is $~ 9 \% \mathrm{ML} \mathrm{Cr}$.

DFT calculations were carried out by using the projector augmented wave method ${ }^{40,41}$ and the generalized gradient approximation with Perdew-Burke-Ernzerhof type functional, ${ }^{42}$ as implemented in the Vienna ab initio simulation package. ${ }^{43}$ Plane wave basis set with a kinetic energy cutoff of $340 \mathrm{eV}$ was used. For the geometrical and electronic structures, a $3 \times 3 \times 1$ supercell slab model with the in-plane experimental lattice constants was constructed, which contained 20 atomic layers (4 $\mathrm{QL}$ ) of $\mathrm{Bi}_{2} \mathrm{Se}_{3}$, absorbed $\mathrm{Cr}$ atoms, and a vacuum layer larger than $20 \AA$ along the $z$ direction. During the structure optimization, 16 atomic layers of $\mathrm{Bi}_{2} \mathrm{Se}_{3}$ on the bottom were fixed and the other atoms were fully relaxed until the residual 
force less than $0.01 \mathrm{eV} / \AA ̊$. The Monkhorst-Pack $k$ points were $2 \times 2 \times 1$. SOC was included to calculate the electronic structure. $^{44}$ For the climbing nudged elastic band (NEB) calculations, ${ }^{33}$ the $3 \times 3 \times 1$ supercell slab model contained 5 atomic layers $(1 \mathrm{QL})$ of $\mathrm{Bi}_{2} \mathrm{Se}_{3}$, absorbed $\mathrm{Cr}$ atoms and a vacuum layer with fully relaxation.

\section{AUTHOR INFORMATION}

\section{Corresponding Authors}

*E-mail: dwh@phys.tsinghua.edu.cn. Phone: +86 010 62772780.

*E-mail: syzhou@mail.tsinghua.edu.cn. Phone: +86 010 62797928.

\section{Author Contributions}

These authors contribute equally to this work.

\section{Notes}

The authors declare no competing financial interest.

\section{ACKNOWLEDGMENTS}

This work is supported by the National Natural Science Foundation of China (Grant Nos. 11274191 and 11334006) and Ministry of Education of China (20121087903, 20121778394). E.Y.W. acknowledges support from the Advanced Light Source doctoral fellowship program. The Advanced Light Source is supported by the Director, Office of Science, Office of Basic Energy Sciences, of the U.S. Department of Energy under Contract No. DE-AC0205CH11231. The crystal growth work at Purdue was supported by the DARPA MESO program (Grant No. N66001-11-14107)

\section{REFERENCES}

(1) Hasan, M. Z.; Kane, C. L. Rev. Mod. Phys. 2010, 82, 3045-3067.

(2) Qi, X.-L.; Zhang, S.-C. Rev. Mod. Phys. 2011, 83, 1057-1110.

(3) Roushan, P.; Seo, J.; Parker, C. V.; Hor, Y.; Hsieh, D.; Qian, D.; Richardella, A.; Hasan, M. Z.; Cava, R.; Yazdani, A. Nature 2009, 460, $1106-1109$.

(4) Zhang, T.; Cheng, P.; Chen, X.; Jia, J.-F.; Ma, X.; He, K.; Wang, L.; Zhang, H.; Dai, X.; Fang, Z.; Xie, X.; Xue, Q.-K. Phys. Rev. Lett. 2009, 103, 266803.

(5) He, L.; Xiu, F.; Yu, X.; Teague, M.; Jiang, W.; Fan, Y.; Kou, X.; Lang, M.; Wang, Y.; Huang, G.; Yeh, N.-C.; Wang, K. L. Nano Lett. 2012, 12, 1486-1490.

(6) Yan, Y.; Liao, Z.-M.; Ke, X.; van Tendeloo, G.; Wang, Q.; Sun, D.; Yao, W.; Zhou, S.; Zhang, L.; Wu, H.-C.; Yu, D.-P. Nano Lett. 2014, 14, 4389-4394.

(7) Chen, Y. L.; Chu, J.-H.; Analytis, J. G.; Liu, Z. K.; Igarashi, K.; Kuo, H.-H.; Qi, X. L.; Mo, S. K.; Moore, R. G.; Lu, D. H.; Hashimoto, M.; Sasagawa, T.; Zhang, S. C.; Fisher, I. R.; Hussain, Z.; Shen, Z. X. Science 2010, 329, 659-662.

(8) Yu, R.; Zhang, W.; Zhang, H.-J.; Zhang, S.-C.; Dai, X.; Fang, Z. Science 2010, 329, 61-64.

(9) Chang, C.-Z.; Zhang, J.; Feng, X.; Shen, J.; Zhang, Z.; Guo, M.; Li, K.; Ou, Y.; Wei, P.; Wang, L.-L.; Ji, Z.-Q.; Feng, Y.; Ji, S.; Chen, X.; Jia, J.; Dai, X.; Fang, Z.; Zhang, S.-C.; He, K.; Wang, Y.; Lu, L.; Ma, X.C.; Xue, Q.-K. Science 2013, 340, 167-170.

(10) Kou, X.; Guo, S.-T.; Fan, Y.; Pan, L.; Lang, M.; Jiang, Y.; Shao, Q.; Nie, T.; Murata, K.; Tang, J.; Wang, Y.; He, L.; Lee, T.-K.; Lee, W.L.; Wang, K. L. Phys. Rev. Lett. 2014, 113, 137201.

(11) Checkelsky, J. G.; Yoshimi, R.; Tsukazaki, A.; Takahashi, K. S.; Kozuka, Y.; Falson, J.; Kawasaki, M.; Tokura, Y. Nat. Phys. 2014, 10, 731-736.

(12) Qi, X.-L.; Hughes, T. L.; Zhang, S.-C. Phys. Rev. B 2008, 78, 195424.
(13) Zhao, L.; Tang, P.; Gu, B.-L.; Duan, W. Phys. Rev. Lett. 2013, $111,116601$.

(14) Zhang, J.-M.; Zhu, W.; Zhang, Y.; Xiao, D.; Yao, Y. Phys. Rev. Lett. 2012, 109, 266405.

(15) Chang, C.-Z.; Tang, P.; Wang, Y.-L.; Feng, X.; Li, K.; Zhang, Z.; Wang, Y.; Wang, L.-L.; Chen, X.; Liu, C.; Duan, W.; He, K.; Ma, X.-C.; Xue, Q.-K. Phys. Rev. Lett. 2014, 112, 056801.

(16) Cha, J. J.; Williams, J. R.; Kong, D.; Meister, S.; Peng, H.; Bestwick, A. J.; Gallagher, P.; Goldhaber-Gordon, D.; Cui, Y. Nano Lett. 2010, 10, 1076-1081.

(17) Cha, J. J.; Claassen, M.; Kong, D.; Hong, S. S.; Koski, K. J.; Qi, X.-L.; Cui, Y. Nano Lett. 2012, 12, 4355-4359.

(18) Wray, L. A.; Xu, S.-Y.; Xia, Y.; Hsieh, D.; Fedorov, A. V.; San Hor, Y.; Cava, R. J.; Bansil, A.; Lin, H.; Hasan, M. Z. Nat. Phys. 2011, 7, 32-37.

(19) Honolka, J.; Khajetoorians, A. A.; Sessi, V.; Wehling, T. O.; Stepanow, S.; Mi, J.-L.; Iversen, B. B.; Schlenk, T.; Wiebe, J.; Brookes, N. B.; Lichtenstein, A. I.; Hofmann, P.; Kern, K.; Wiesendanger, R. Phys. Rev. Lett. 2012, 108, 256811.

(20) Zhang, J.; Chang, C.-Z.; Tang, P.; Zhang, Z.; Feng, X.; Li, K.; Wang, L.-1.; Chen, X.; Liu, C.; Duan, W.; He, K.; Xue, Q.-K.; Ma, X.; Wang, Y. Science 2013, 339, 1582-1586.

(21) Corliss, L. M.; Elliott, N.; Hastings, J. M.; Sass, R. L. Phys. Rev. 1961, 122, 1402-1406.

(22) Boeglin, C.; Ohresser, P.; Decker, R.; Bulou, H.; Scheurer, F.; Chado, I.; Dhesi, S. S.; Gaudry, E.; Lazarovits, B. Phys. Status Solidi B 2005, 242, 1775-1778.

(23) Huang, D. J.; Jeng, H.-T.; Chang, C. F.; Guo, G. Y.; Chen, J.; Wu, W. P.; Chung, S. C.; Shyu, S. G.; Wu, C. C.; Lin, H.-J.; Chen, C. T. Phys. Rev. B 2002, 66, 174440.

(24) King, P. D. C.; Hatch, R. C.; Bianchi, M.; Ovsyannikov, R.; Lupulescu, C.; Landolt, G.; Slomski, B.; Dil, J. H.; Guan, D.; Mi, J. L.; Rienks, E. D. L.; Fink, J.; Lindblad, A.; Svensson, S.; Bao, S.; Balakrishnan, G.; Iversen, B. B.; Osterwalder, J.; Eberhardt, W.; Baumberger, F.; Hofmann, P. Phys. Rev. Lett. 2011, 107, 096802.

(25) Benia, H. M.; Lin, C.; Kern, K.; Ast, C. R. Phys. Rev. Lett. 2011, 107, 177602.

(26) Valla, T.; Pan, Z.-H.; Gardner, D.; Lee, Y. S.; Chu, S. Phys. Rev. Lett. 2012, 108, 117601.

(27) Luttinger, J. Phys. Rev. 1960, 119, 1153-1163.

(28) LaShell, S.; McDougall, B. A.; Jensen, E. Phys. Rev. Lett. 1996, $77,3419-3422$.

(29) Nechaev, I. A.; Jensen, M. F.; Rienks, E. D. L.; Silkin, V. M.; Echenique, P. M.; Chulkov, E. V.; Hofmann, P. Phys. Rev. B 2009, 80, 113402 .

(30) Nitta, J.; Akazaki, T.; Takayanagi, H.; Enoki, T. Phys. Rev. Lett. 1997, 78, 1335-1338.

(31) Grundler, D. Phys. Rev. Lett. 2000, 84, 6074-6077.

(32) $E_{\mathrm{b}}$ is defined as $\left(E_{\mathrm{t}}-E_{\mathrm{s}}-n \mu_{\mathrm{Cr}}\right) / n$, where $E_{\mathrm{t}}$ is the total energy of whole system, $E_{\mathrm{s}}$ and $\mu_{\mathrm{Cr}}$ are the energy of intrinsic $\mathrm{Bi}_{2} \mathrm{Se}_{3}$ thin film and the chemical potential of $\mathrm{Cr}$ atom respectively, and $n$ is the number of $\mathrm{Cr}$ atoms.

(33) (a) Henkelman, G.; Uberuaga, B. P.; Jónsson, H. J. Chem. Phys. 2000, 113, 9901-9904. (b) Henkelman, G.; Jonsson, H. J. Chem. Phys. 2000, 113, 9978-9985.

(34) Bruno, P. Phys. Rev. B 1989, 39, 865-868.

(35) Daalderop, G. H. O.; Kelly, P. J.; Schuurmans, M. F. H. Phys. Rev. B 1994, 50, 9989-10003.

(36) Here, below the Fermi level, the states of $\mathrm{Cr}$ are mainly contributed by spin-majority $E_{2}^{\uparrow}$ states; above the Fermi level, the states of $\mathrm{Cr}$ are mainly contributed by spin-minority $E_{2}^{\downarrow}$ states and spinmajority $E_{1}^{\uparrow}, A_{1}^{\uparrow}$ states. The coupling of $E_{2}^{\uparrow}$ with $E_{2}^{\downarrow}$ and $E_{1}^{\uparrow}$ results in an in-plane magnetic easy axis; the coupling of $E_{2}^{\uparrow}$ with $A_{1}^{\uparrow}$ is zero.

(37) Liu, X.; Hsu, H.-C.; Liu, C.-X. Phys. Rev. Lett. 2013, 111, 086802 .

(38) Kuroda, K.; Arita, M.; Miyamoto, K.; Ye, M.; Jiang, J.; Kimura, A.; Krasovskii, E. E.; Chulkov, E. V.; Iwasawa, H.; Okuda, T.; Shimada, K.; Ueda, Y.; Namatame, H.; Taniguchi, M. Phys. Rev. Lett. 2010, 105, 076802 . 
(39) Henkelman, G.; Arnaldsson, A.; Jónsson, H. Comput. Mater. Sci. 2006, 36, 354-360.

(40) Blöchl, P. E. Phys. Rev. B 1994, 50, 17953-17979.

(41) Kresse, G.; Joubert, D. Phys. Rev. B 1999, 59, 1758-1775.

(42) Perdew, J. P.; Burke, K.; Ernzerhof, M. Phys. Rev. Lett. 1996, 77, 3865-3868.

(43) Kresse, G.; Furthmüller, J. Phys. Rev. B 1996, 54, 11169-11186.

(44) Hobbs, D.; Kresse, G.; Hafner, J. Phys. Rev. B 2000, 62, 1155611570 . 